Pacific Journal of Mathematics

PROJECTIVE LATTICES

Prese and James Bryant Nation 


\title{
PROJECTIVE LATTICES
}

\author{
Ralph Freese and J. B. Nation
}

\begin{abstract}
This paper gives necessary and sufficient conditions for a lattice to be projective. The conditions are the Whitman condition, and a condition of Jónsson, and two new conditions explained below.
\end{abstract}

We begin with several definitions. First, a lattice $L$ is projective if for any lattices $M, N$ and any lattice homomorphism $h$ of $L$ into $N$ and $f$ of $M$ onto $N$, there is a homomorphism $g$ of $L$ into $M$ such that $f(g(a))=h(a)$ for all $a \in L$. This is equivalent to the condition that there is a homomorphism $f$ of a free lattice $F L(X)$ onto $L$ and a homomorphism $g$ of $L$ into $F L(X)$ such that $f(g(a))=a$ for all $a \in L$. The map $f \circ g$ is a retraction, i.e., it is an endomorphism of $F L(X)$ which is pointwise-fixed on its image. The image of a retraction is called a retract. Thus the class of projective lattices coincides with the class of retracts of free lattices. Since lattice epimorphisms, in the categorical sense, are precisely onto homomorphisms, [11, p. 150-151] the above definition of projective lattices agrees with the categorical one.

If $a$ is an element of a lattice $L$ and $U$ is a finite, nonempty subset of $L$ such that $a \leqq \vee U$, then $U$ is a cover of $a$. A cover $U$ of $a$ is trivial if $a \leqq u$ for some $u \in U$. If $U$ and $V$ are finite nonempty subsets of $L$ we write $U \ll V$ if for each $u \in U$ there is a $v \in V$ with $u \leqq v$. A cover $U$ of $a$ is called a minimal cover of $a$ if whenever $V$ is a cover of $a$ such that $V \ll U$, then $U \subseteq V$. We let $(W)$ denote the Whitman condition: for all $a, b, c, d, a \wedge b \leqq c \vee d$ implies either $a \wedge b \leqq c, a \wedge b \leqq d, a \leqq c \vee d$, or $b \leqq c \vee d$. Let $f$ be a map from $K$ onto $L$; then a map $g$ from $L$ into $K$ is called a transversal of $f$ if $f(g(a))=a$ for all $a \in L$. L is a bounded homomorphic image of a free lattice if there is a homomorphism $f$ mapping a free lattice, $F L(X)$, onto $L$ such that $\{w \in F L(X): f(w)=a\}$ has a least and greatest element for each $a \in L$. This concept originated with R. McKenzie and a theorem of Jónsson, Kostinsky, and McKenzie $[16,17]$ states that a finitely generated lattice is projective if and only if it is a bounded homomorphic image of a free lattice and satisfies $(W)$.

B. Jónsson, in his studies of sublattices of free lattices, defined $D_{0}(L)$ to be the set of join-prime elements of $L$ ( $a \in L$ is join-prime if $a \leqq b \vee c$ implies $a \leqq b$ or $a \leqq c)$. $\quad D_{k+1}(L)$ is the set of elements $a$ of $L$ such that if $U$ is a nontrivial cover of $a$ then there is a $V \subseteq D_{k}(L)$ such that $V$ is a 
cover of $a$ and $V \ll U$. The set $D_{k}^{\prime}(L)$ is defined dually. Jónsson's condition is $\bigcup_{k<\omega} D_{k}(L)=L=\bigcup_{k<\omega} D_{k}^{\prime}(L)$. Although McKenzie's notion of a bounded homomorphic image of a free lattice and Jónsson's condition are quite different in appearance, they are actually the same for finitely generated lattices. This fact was hinted at by McKenzie in his limit tables [17], proved for finite lattices by H. S. Gaskill and C. R. Platt [10], and proved for finitely generated lattices by B. Jónsson and J. B. Nation [15]. McKenzie's condition is simpler and more pictorial; Jónsson's is internally defined and one can effectively, and even efficiently, decide if a finite lattice satisfies it.

McKenzie's and Jónsson's conditions are not the same for lattices in general. Indeed, $\omega=\{0,1,2, \cdots\}$ under its natural ordering has $D_{0}(\omega)=\omega$, but it is not a bounded homomorphic image of a free lattice. We shall show that every projective lattice satisfies Jónsson's condition.

The length of a lattice term (or word) $t$ is the number of times the symbols $\vee$ and $\wedge$ occur in $t$. P. Whitman showed that corresponding to each element $a$ of a free lattice $F L(X)$, there is a term $t$ of minimal length representing $a$, and that $t$ is unique up to commutivity and associativity. Furthermore, he showed that the term $t$ was closely connected to the arithmetic of $F L(X)$, by showing that if $t=t_{1} \vee \cdots \vee t_{n}$, where each $t_{t}$ is not of the form $r \vee s$, and $a=\vee U$, then $\left\{a_{1}, \cdots, a_{n}\right\} \ll U$, where $a_{i}$ is the element of $F L(X)$ corresponding to $t_{i}$. It has become apparent that in the study of projective lattices and sublattices of free lattices we need to understand not only the equations $a=\vee U$ but also $a \leqq \vee U$, that is, the covers of $a$. In this regard we generalize Whitman's result by showing that for each $a \in F L(X)$ there is a finite set, $S(a)$, of nontrivial, minimal covers of $a$, such that if $U$ is a nontrivial cover of $a$, then there is a $V \in S(a)$ with $V \ll U$. $\quad S(a)$ may be formed as follows. Let $t$ be the term of minimal length corresponding to $a$, and let $T$ be the set of elements of $F L(X)$ corresponding to the subterms of $t$. Then $S(a)$ is the set of nontrivial, minimal covers of $a$ whose elements lie in $T$. This condition of having a finite set of minimal covers of $a$ is preserved under retracts, and hence, holds in projective lattices. This condition and its dual, together with Jónsson's condition and $(W)$, characterize countable projective lattices.

In the uncountable case we need a fourth condition. We require that some epimorphism of a free lattice onto $L$ have an order-preserving transversal. Any countable lattice satisfies this condition [5], and any uncountable chain fails it, but satisfies the three other conditions above. Indeed, Galvin and Jónsson have shown that $F L(X)$ contains no uncountable chains [9]. This fourth condition is equivalent to the following more satisfactory condition: for each $a \in L$ there are two finite 
subsets $A(a) \subseteq\{c \in L: c \geqq a\}$ and $B(a) \subseteq\{c \in L: c \leqq a\}$ such that if $a \leqq b$, then $A(a) \cap B(b) \neq \varnothing$.

The main result of this paper is the following theorem.

THEOREM 1. A lattice is projective if and only if it satisfies each of the following conditions.

(1) $(W)$

(2) $\bigcup_{k<\omega} D_{k}(L)=L=\bigcup_{k<\omega} D_{k}^{\prime}(L)$

(3) for each $a \in L$, there is a finite set $S(a)$ of nontrivial covers of a, such that, if $U$ is any nontrivial cover of $a$, then $V \ll U$ for some $V \in S(a)$

(3') the dual of (3)

(4) for each $a \in L$, there are two finite subsets, $A(a) \subseteq\{c \in L: c \geqq$ $a\}$ and $B(a) \subseteq\{c \in L: c \leqq a\}$ such that, if $a \leqq b$, then $A(a) \cap B(b) \neq \varnothing$.

Corollary. A countable lattice is projective if and only if it satisfies (1), (2), (3), and (3').

The first section of this paper shows that (1)-(4) hold in projective lattice. This is the more difficult part of the proof. The second section gives the proof that (1)-(4) are sufficient. The third section gives some examples showing that the above conditions are independent. Other properties of projective lattices are discussed and shown to be insufficient to characterize projective lattices. For example, condition (4) of Theorem 1 cannot be replaced with " $L$ has no uncountable chains." Indeed, it is shown that the ordinal sum of two uncountable free lattices is not projective (although it is a sublattice of a free lattice).

The final section discusses projective distributive lattices and projective Boolean algebras. A slight generalization of the characterization of projective distributive lattices of Balbes and Horn $[2,4]$ is given.

The authors would like to thank Professor George Grätzer for some suggestions which shortened the proofs of Lemma 4 and Theorem 2.

1. Necessity. In this section we show that (1)-(4) hold in projective lattices. We begin with two lemmas about free lattices, the first of which was proved in [15]. Let $S$ be the join-closure operator; that is, if $\boldsymbol{A}$ is a subset of a lattice, then $\boldsymbol{S}(\boldsymbol{A})$ is the set of all finite, nonempty joins of elements of $\boldsymbol{A}$. $\boldsymbol{P}$ is defined dually.

Lemma $1 . \quad D_{k}(F L(X))=(\boldsymbol{P S})^{k} \boldsymbol{P}(X)$.

LEMMA 2. $F L(X)$ satisfies condition (3).

Proof. If $a \in D_{0}(F L(X))$, then $a$ is join-prime and thus has no 
nontrivial covers. In this case we set $S(a)=\varnothing$. Now suppose $a \in D_{k}(F L(X)), k>0$. By Lemma 1, there are finite subsets $V_{i} \subseteq$ $D_{k-1}(F L(X)), \quad i=1, \cdots, r, \quad$ such that $a=\wedge_{i=1}^{r}\left(\vee V_{l}\right) \quad(r=1 \quad$ is allowed). Let $T=\{b \in F L(X)$ : for some $i \in\{1, \cdots, r\}$, and some $v \in$ $V_{i}$, and some $\left.U \in S(v), b \in U\right\} \cup \cup_{i=1}^{r} V_{i}$. It follows from an easy induction on $k$ that $T$ is finite. Let $S(a)$ be the set of all subsets of $T$ which are nontrivial covers of $a$. Since $T$ is finite, $S(a)$ is finite. Suppose $U$ is a nontrivial cover of $a$. Then $a=\wedge_{i=1}^{r}\left(\vee V_{i}\right) \leqq$ $\vee U$. From $(W)$ and the fact that $U$ is a nontrivial cover of $a$, we conclude that for some $i$ and all $v \in V_{i}, v \leqq \vee U$. If $U$ is a nontrivial cover of $v$ then, since $v \in D_{k-1}(F L(X))$, there is, by induction on $k$, $U_{v} \in S(v)$ with $U_{v} \ll U$. If $v \leqq u$ for some $u \in U$, we set $U_{v}=$ $\{v\}$. Let $U_{0}=\cup_{v \in V_{i}} U_{v}$. Clearly $U_{0} \ll U$. Also, since $\vee U_{v} \geqq v$, for each $v \in V_{i}, \vee U_{0} \geqq \vee V_{i} \geqq a$. Thus $U_{0} \subseteq T, U_{0}$ is a cover of $a$, and, since $U_{0} \ll U, U_{0}$ is a nontrivial cover of $a$. Hence $U_{0} \in S(a)$, proving the lemma.

An easy induction shows that the set $T$ constructed above is contained in $D_{k-1}(F L(X))$. Thus if $U \in S(a), a \in D_{k}(F L(X))$, then $U \subseteq D_{k-1}(F L(X))$. This fact will be used in Lemma 4. Also note that we can delete the nonminimal members of $S(a)$ and the resulting set still satisfies (3).

LEMMA 3. If $L$ is a projective lattice, then $L$ satisfies (3).

Proof. Let $f: F L(X) \rightarrow L$ be an epimorphism, and $g: L \rightarrow F L(X)$ be a homomorphism such that $f(g(a))=a$ for all $a \in L$. For each $a \in L$, let $S(a)=\{U \subseteq L: U$ is a nontrivial cover of $a$ and $U \subseteq f(V)$ for some $V \in S(g(a))\}$. Let $U$ be an arbitrary nontrivial cover of $a$. Then $g(U)$ is a nontrivial cover of $g(a)$. Hence there is a $V \in S(g(a))$ with $V \ll g(U)$. Consequently, $f(V)$ covers $a$ and $f(V) \ll f(g(U))=$ $U$. Since $U$ is a nontrivial cover of $a$, the above implies that $f(V)$ is a nontrivial cover of $a$. Hence $f(V) \in S(a)$, proving the lemma.

LEMMA 4. If $L$ is projective, then $\cup_{k<\omega} D_{k}(L)=L$.

Proof. Let $f: F L(X) \rightarrow L$ be an epimorphism, and $g: L \rightarrow F L(X)$ be a homomorphism such that $f(g(a))=a$, for all $a \in L$. By induction on $k$, we shall show that for all $b \in F=F L(X)$

$$
b \in D_{k}(F) \text { and } \quad g f(b) \geqq b \quad \text { imply } \quad f(b) \in D_{k}(L) .
$$

The lemma follows from this, since if $a \in L$, then we may choose 
$b=g(a)$. Then $g(f(b))=g(f(g(a)))=g(a)=b$. Hence $a \in D_{k}(L)$, where $k$ is such that $g(a) \in D_{k}(F)$.

Suppose $b \in D_{k}(F)$ and $g(f(b)) \geqq b$. Let $U$ be a cover of $f(b)$. Then $g(U)$ is a cover of $g(f(b))$. Since $g(f(b)) \geqq b, g(U)$ is a cover of $b$. If $g(u) \geqq b$ for some $u \in U$, then $u=f(g(u)) \geqq$ $f(b)$. Hence, if $U$ is a nontrivial cover of $f(b)$, then $g(U)$ is a nontrivial cover of $b$. In particular, if $b \in D_{0}(F)$ then $f(b) \in D_{0}(L)$. Now suppose that $U$ is a nontrivial cover of $f(b)$. It follows from Lemma 3 that there is a minimal, nontrivial cover $U^{\prime}$ of $f(b)$ with $U^{\prime} \ll U$. Then $g\left(U^{\prime}\right)$ is a nontrivial cover of $b$, and by Lemma 2 there is a $U_{0} \in S(b)$ with $U_{0} \ll g\left(U^{\prime}\right)$. Now $f\left(U_{0}\right)$ is a cover of $f(b)$ and $f\left(U_{0}\right) \ll f\left(g\left(U^{\prime}\right)\right)=$ $U^{\prime}$. Since $U^{\prime}$ is minimal $U^{\prime} \subseteq f\left(U_{0}\right)$. Thus $U_{0} \ll g\left(U^{\prime}\right) \subseteq$ $g f\left(U_{0}\right)$. Hence $U_{0} \ll g\left(f\left(U_{0}\right)\right)$.

Let $U_{0}=\left\{u_{1}, \cdots, u_{r}\right\}$. Since $U_{0} \ll g\left(f\left(U_{0}\right)\right)$, there is a function $\sigma$ from $\{1, \cdots, r\}$ into itself such that $u_{j} \leqq g\left(f\left(u_{\sigma(j)}\right)\right), j=1, \cdots, r$. Applying $f$, we obtain $f\left(u_{j}\right) \leqq f\left(g\left(f\left(u_{\sigma(j)}\right)\right)\right)=f\left(u_{\sigma(j)}\right)$. Consider the sequence $1=$ $\sigma^{0}(1), \sigma(1), \sigma^{2}(1), \sigma^{3}(1), \cdots . \quad$ For some $s<t, \sigma^{s}(1)=\sigma^{t}(1)$ and $1, \sigma(1)$, $\sigma^{2}(1), \cdots, \sigma^{s}(1)$ are distinct. Then

$$
f\left(u_{\sigma^{s}(1)}\right) \leqq f\left(u_{\sigma^{s+1}(1)}\right) \leqq \cdots \leqq f\left(u_{\sigma^{\prime}(1)}\right)=f\left(u_{\sigma^{s}(1)}\right)
$$

Hence all of these inequalities must be equalities. Thus

$$
u_{\sigma^{s}(1)} \leqq g\left(f\left(u_{\sigma^{s+1}(1)}\right)\right)=g\left(f\left(u_{\sigma^{s}(1)}\right)\right)
$$

and similarly,

$$
u_{\sigma^{s+1}(1)} \leqq g\left(f\left(u_{\sigma^{s+1}(1)}\right)\right), \cdots, u_{\sigma^{t-1}(1)} \leqq g\left(f\left(u_{\sigma^{t-1}(1)}\right)\right)
$$

and moreover

$$
f\left(u_{1}\right) \leqq f\left(u_{\sigma(1)}\right) \leqq f\left(u_{\sigma^{2}(1)}\right) \leqq \cdots \leqq f\left(u_{\sigma^{s}(1)}\right) .
$$

Hence $f\left(U_{0}-\left\{u_{1}, u_{\sigma(1)}, \cdots, u_{\sigma^{s-1}(1)}\right\}\right)$ still covers $f(b)$. These arguments show that if we let $U_{0}^{\prime}=\left\{u \in U_{0}: u \leqq g(f(u))\right\}$, then $f\left(U_{0}^{\prime}\right)$ is a cover of $f(b)$. By the remark following Lemma $2, U_{0} \subseteq D_{k-1}(F)$. By induction we have $f(u) \in D_{k-1}(L)$ for each $u \in U_{0}^{\prime}$. Since $f\left(U_{0}\right) \ll U, f\left(U_{0}^{\prime}\right) \ll$ $U$. Hence $f(b) \in D_{k}(L)$, completing the proof.

These lemmas show that a retract of a lattice satisfying (2) and (3) again satisfies (2) and (3). It is interesting that there are lattices which satisfy (2) which have retracts that do not satisfy (2).

LeMma 5. $F L(X)$ satisfies (4). 
Proof. If $a \in F L(X)$ let $\operatorname{var}(a) \subseteq X$ be those variables occurring in the cannonical (shortest) expression for $a$. If $a \in D_{k}(F L(X))$ $D_{k-1}(F L(X))$ and $b \in D_{l}^{\prime}(F L(X))-D_{l-1}^{\prime}(F L(X))$ set

$$
\begin{aligned}
& A(a)=\left((\boldsymbol{P S})^{k} \boldsymbol{P}(\operatorname{var} a)\right) \cap 1 / a \\
& \boldsymbol{B}(\boldsymbol{b})=\left((\boldsymbol{S P})^{\prime} \boldsymbol{S}(\operatorname{var} b)\right) \cap b / 0
\end{aligned}
$$

where in general $1 / a$ is the set of all elements greater than or equal to $a$, even if the lattice does not have a $1 ; a / 0$ is defined dually.

We show by induction on the pair $(k, l)$ that if $a \leqq b$ in $F L(X)$ then $A(a) \cap B(b) \neq \varnothing$. We leave the case $k=l=0$ to the reader. Suppose $k=0$ and $l>0$. Then $a \in D_{0}(F L(X))$ in join-prime and $b=\vee_{i=1}^{n} \wedge U_{i}, U_{i} \subseteq D_{i-1}^{\prime}(F L(X))$, by the dual of Lemma 1. Thus $a \leqq \wedge U_{i}$ for some $i$. Hence $a \leqq u$ for all $u \in U_{i}$. However, $U_{i} \subseteq$ $D_{l-1}^{\prime}(F L(X))$. Hence by induction there is a $c_{u} \in A(a) \cap B(u)$. Since $c_{u} \geqq a$ for each $u \in U_{i}, c=\wedge c_{u} \geqq a$. Since $A(a)$ is meet-closed, $c \in A(a)$. Since $\quad \operatorname{var}(u) \subseteq \operatorname{var}(b), \quad B(u)=(\boldsymbol{S P})^{l-1} \boldsymbol{S}(\operatorname{var} u) \subseteq$ $(\boldsymbol{S P})^{l-1} \boldsymbol{S}(\operatorname{var} b)$. It follows that $c=\wedge c_{u}$ is in $B(b)$.

Now suppose $k>0, l>0$. Then

$$
a=\wedge_{i=1}^{m}\left(\wedge V_{i}\right) \leqq \vee_{i=1}^{n}\left(\wedge U_{i}\right)=b
$$

By $(W)$ either there is an $i$ such that for all $v \in V_{i}, v \leqq b$, or the dual situation holds. In the former case induction yields that there is a $c_{v} \in A(v) \cap B(b)$. Let $c=\vee\left(c_{v}: v \in V_{\imath}\right)$. Then $c \in A(a) \cap B(b)$. The proof of the lemma is completed by duality.

THEOREM 2. Condition (4) of Theorem 1 holds in a lattice L if and only if

(*) there is a homomorphism $f$ from a free lattice $F$ onto $L$ and an order preserving map $g: L \rightarrow F$ such that $f(g(a))=a$ for all $a \in L$.

Proof. We leave it for the reader to show that if $(*)$ holds for some homomorphism of a free lattice onto $L$, it holds for all such homomorphisms. Thus in proving the theorem we shall take $X=$ $\left\{x_{a}: a \in L\right\}$ and $f: F L(X) \rightarrow L$ to be the extension of $f\left(x_{a}\right)=a$.

Suppose $L$ satisfies (*). For $a \in L$ set $A(a)=f(A(g(a)))$ and $B(a)=f(B(g(a)))$. If $a \leqq b$ in $L$, then $g(a) \leqq g(b)$. Hence $A(g(a)) \cap B(g(b)) \neq \varnothing$. Thus $A(a) \cap B(b) \neq \varnothing$.

Now suppose that $L$ satisfies (4). We arrange $L$ into a wellordered, doubly indexed sequence as follows. Choose $a_{00} \in L$. Let $a_{00}$, 
$a_{01}, \cdots, a_{0 n}$ be $A\left(a_{00}\right) \cup B\left(a_{00}\right) \cup\left\{a_{00}\right\}$ in some order. Let $a_{0, n+1}, \cdots, a_{0, n+m}$ be $A\left(a_{01}\right) \cup B\left(a_{01}\right)-\left\{a_{00}, \cdots, a_{0 n}\right\}$. Continuing in this way we obtain a countable or finite sequence $a_{00}, a_{01}, a_{02}, \cdots$. If this exhausts $L$, we stop. Otherwise, choose $a_{10} \in L-\left\{a_{00}, a_{01}, \cdots\right\}$. Now repeat the above process with $a_{10}$, except, of course, omit any element which has occurred previously. Eventually, we obtain $L=$ $\left(a_{\alpha i}: \alpha<\gamma, i<\mu_{\alpha}\right)$ for some ordinals $\gamma$ and $\mu_{\alpha} \leqq \omega$. This sequence has the property that if $b \in A(a) \cup B(a)$, then either $b$ comes before $a$ in the sequence, or, at worse, only finitely many places after $a$. Define inductively $g: L \rightarrow F L(X)$ by

$$
\begin{aligned}
g\left(a_{\alpha i}\right)=\{ & x_{a_{\alpha i}} \wedge \wedge\left\{g\left(a_{\alpha j}\right): j<i, a_{\alpha \jmath}>a_{\alpha i}\right\} \\
& \left.\wedge\left\{g\left(a_{\beta J}\right): \beta<\alpha, j<\mu_{\beta}, a_{\beta j} \in A\left(a_{\alpha i}\right)\right\}\right\} \\
& \vee\left\{g\left(a_{\alpha j}\right): j<i, a_{\alpha J}<a_{\alpha i}\right\} \vee \\
& \vee\left\{g\left(a_{\beta j}\right): \beta<\alpha, j<\mu_{\beta}, a_{\beta j} \in B\left(a_{\alpha i}\right)\right\} .
\end{aligned}
$$

(Empty joins or meets are simply omitted.) Straightforward inductions show that $g$ is a transversal of $f$ and that $g$ is order-preserving.

Finally, note that every countable lattice satisfies (4) (cf. [5]). If $L=\left\{a_{0}, a_{1}, \cdots\right\}$ then set $A\left(a_{n}\right)=\left\{a_{i}: i \leqq n\right.$ and $\left.a_{i} \geqq a_{n}\right\}$ and define $B\left(a_{n}\right)$ dually.

2. Sufiiciency. The proof of the sufficiency is essentially the same as [15]. We sketch the details here. Let $f$ be a homomorphism of a free lattice onto a lattice $L$ which satisfies (1)-(4). By Theorem $2, f$ has an order-preserving transversal $g$. Define $g_{0}=g$ and

$$
g_{k+1}(a)=g(a) \wedge \wedge\left\{\vee g_{k}(U): U \in S(a)\right\}
$$

By an easy induction, if $a \in D_{k}(L)$, then $g_{k}(a)=g_{m}(a)$ for all $m \geqq$ $k$. Hence the sequence $\left\{g_{0}(a), g_{1}(a), \cdots\right\}$ is eventually constant. Let $g_{-}(a)$ denote this final value. Since $g$ is order-preserving, each $g_{k}$ and $g_{-}$is also. Furthermore, $g_{-}$, preserves joins. To see this let $a$ and $b$ be incomparable elements of $L$ and choose $k$ large enough so that $g_{k}(a)=$ $g_{-}(a), g_{k}(b)=g_{-}(b)$, and $g_{k}(a \vee b)=g_{-}(a \vee b)$. Since $\{a, b\}$ is a nontrivial cover of $a \vee b$, there is a $U_{0} \in S(a \vee b)$ with $U_{0} \ll\{a, b\}$. Thus $\vee g_{k}\left(U_{0}\right) \leqq g_{k}(a) \vee g_{k}(b)$, from the monotonicity of $g_{k}$. Examining the definition of $g_{k+1}(a \vee b)$ we see that $g_{-}(a \vee b)=g_{k+1}(a \vee b) \leqq \vee g_{k}\left(U_{0}\right) \leqq$ $g_{k}(a) \vee g_{k}(b)=g_{-}(a) \vee g_{-}(b)$. Thus from the monotonicity of $g_{-}$, we see that $g_{-}(a \vee b)=g_{-}(a) \vee g_{-}(b)$.

Since $L$ satisfies $(W)$, every nontrivial cover of $a \wedge b$ is a nontrivial 
cover of $a$ or of $b$. Hence for each $U \in S(a \wedge b)$ there is a $U^{\prime} \in S(a) \cup$ $S(b)$ with $U^{\prime} \ll U$. With the aid of this, the reader can show that if $g$ preserves meets then $g$ - does also.

The proof may now be completed as follows. Let $g^{-}$be the map defined dually to $g_{-}$. Let $h=\left(g^{-}\right)_{-}$. By the dual of the above remarks $g^{-}$preserves meets, and hence $h$ does also. The above remarks also show that $h$ preserves joins. Hence $h$ is a homomorphism of $L$ into $F L(X)$. It is easy to check that $h$ is a transversal of $f$.

3. Examples and discussion. We first give some conditions which hold in projective lattices and present some examples showing that none of these new conditions can replace any of (1)-(4) in Theorem 1. The first condition is a weak form of relative pseudocomplementation.

(5) for each $a, b \in L, a<b$ implies there is a unique $c$ such that $a=b \wedge c$ and such that $a=b \wedge d$ implies $d \leqq c$.

(6) for each $a \in L$, there is a $n(a)<\omega$ such that $a=\vee U$ implies there is a $U^{\prime} \subseteq U,\left|U^{\prime}\right| \leqq n(a)$ with $a=\vee U^{\prime}$.

(7) for each $a \in L$, there is a unique antichain $U$ such that $a=\vee U$ and $a=\vee V$ implies $U \ll V$.

(8) $L$ has no uncountable chains.

Condition (7) is, of course, Whitman's canonical joins [18, 19]. Condition (6) and (8) hold in all sublattices of free lattices, as was

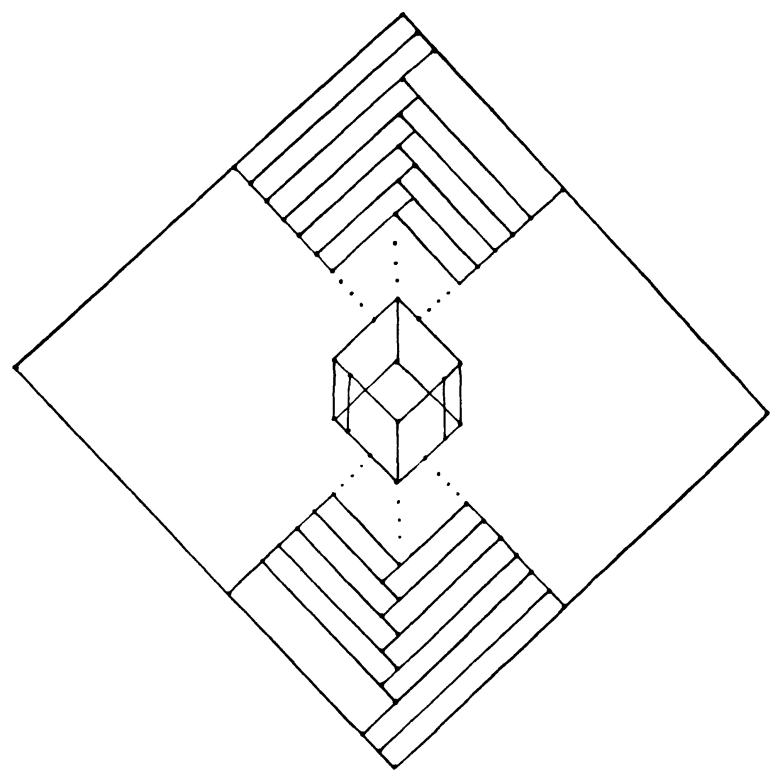

Fig. 1 
shown by Jónsson [13]. Condition (7) is equivalent to the conjunction of (6) and the dual of (5) (cf. [14]). A famous, unsettled conjecture of Jónsson states that a finite lattice is a sublattice of a free lattice if and only if it satisfies $(W),(5)$, and the dual of (5). The infinite analogue of this conjecture is false. The lattice $L_{0}$, diagrammed in Figure 1 , is finitely generated and satisfies (1) and (3)-(8) and their duals, yet fails (2). In particular, (2) cannot be replaced by (5) in Theorem 1. The details of the construction of $L_{0}$ are given in [15].

The lattice $L_{1}$, diagrammed in Figure 2, satisfies (1), (2), (4)-(8), and

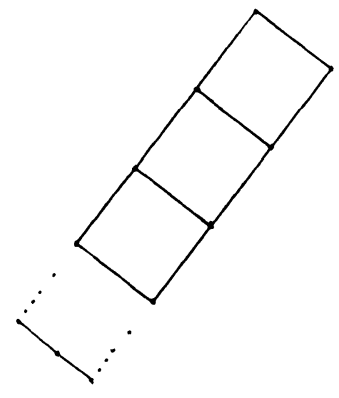

Fig. 2

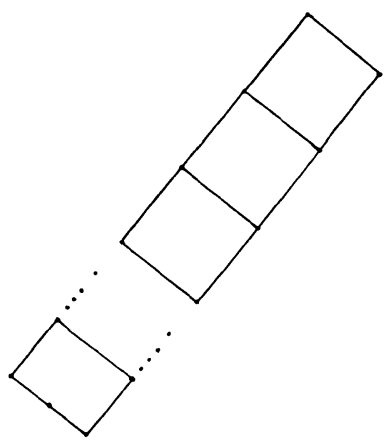

Fig. 3

their duals, but fails (3). Hence $L_{1}$ is not projective. It is, however, a sublattice of a free lattice since it is a sublattice of $L_{2}$, diagrammed in Figure 3, which is projective by the corollary to Theorem 1 . This shows that (1) and (2) alone, are not sufficient to characterize countable projective lattices, even though, by the theorem of Jonsson, Kostinsky, and McKenzie, they do characterize finitely generated projective lattices.

Jónsson and Galvin proved (8) by defining $a \sim b$ in $F L(X)$ if there is an automorphism $\sigma$ of $F L(X)$, generated by a permutation of $X$, with $\sigma(a)=b$. Roughly speaking, $a \sim b$ means $a$ and $b$ are the same word but with the letters changed. They showed that this equivalence relation has only countably many classes, and each class is an antichain (no two elements are comparable). Thus $F L(X)$, and any sublattice of $F L(X)$, is a countable union of antichains. This implies (8). It is actually stronger than (8): there is a partially ordered set with no uncountable chains, which is not a countable union of antichains, [6] p. 5. We give some examples which show that neither (8) nor the stronger condition mentioned above can replace (4) in Theorem 1.

For a partially ordered set $P$, let $F L(P)$ denote the free lattice generated by $P$, i.e., $F L(P)$ is defined by the universal property that any order-preserving map from $P$ to a lattice $L$ can be uniquely extended to a 
homomorphism of $F L(P)$ to $L .^{1} \quad$ A thorough study of these lattices was carried out by R. A. Dean, who showed that they satisfy many of the properties of free lattices. For example, one can decide if $a \leqq b$ in $F L(P)$, by making the same reduction as Whitman's procedure for $F L(X)$. This reduction leads to inequalities of the form $p \leqq q, p$, $q \in P$. The only difference is that in $F L(X), x \leqq y$ if and only if $x=y$; whereas in $F L(P), p \leqq q$ if and only if this holds in $P$. In particular, $F L(P)$ satisfies $(W)$, the elements of $P$ are join- and meet-prime in $F L(P)$, and $F L(P)$ has canonical joins and meets (condition (7) and its dual). Using these facts it is not hard to modify the proof of Theorem 1 to prove the following result.

THEOREM 3. For any partially ordered set $P, F L(P)$ satisfies (1), (2), and (3).

LEMMA 7. $F L(P)$ satisfies (4) if and only if for each $p \in P$ there are two finite sets $A(p) \subseteq\{q \in P: q \geqq p\}$ and $B(p) \subseteq\{q \in P: q \leqq p\}$ such that if $p \leqq q$ in $P$, then $A(p) \cap B(q) \neq \varnothing$.

Proof. Suppose $F L(P)$ satisfies (4). For each $a \in F L(P)$ there is a finite subset $P_{0}$ of $P$ such that $a$ is in the sublattice generated by $P_{0}$. Let $\operatorname{var}(a)$ be such a set. Let $A(a)$ and $B(a)$ be the sets given by (4). For $p \in P$, let $A^{\prime}(p)=\{q \in P: q \geqq p \quad$ and $q \in \operatorname{var}(a)$ for some $a \in A(p)\} . \quad B^{\prime}(p)$ is defined dually. Suppose $p \leqq q$ in $P$. Then, by (4), there is an $a \in A(p) \cap B(q)$. Using the fact that $p$ is join-prime and $q$ is meet-prime, a routine induction on the complexity of $a$ shows that there is an $r \in \operatorname{var}(a)$ with $p \leqq r \leqq q$. Hence $A^{\prime}(p) \cap B^{\prime}(p) \neq \varnothing$.

Conversely, suppose $P$ satisfies the condition of the lemma. Let $X=\left\{x_{p}: p \in P\right\}$ and let $f: F L(X) \rightarrow F L(P)$ be the map extending the map $f\left(x_{p}\right)=p$. Exactly as in the proof of Theorem 2, we can arrange $P$ into a doubly indexed sequence, and, with the aid of this sequence, we can find an order-preserving map $g: P \rightarrow F L(X)$ such that $f(g(p))=p$, for all $p \in P$. By the universal property of $F L(P), g$ can be extended to a homomorphism from $F L(P)$ into $F L(X)$. Clearly this extended map is a transversal of $f$. Thus by Theorem $2, F L(P)$ satisfies (4).

COROLlaRY. For any partially ordered set $P, F L(P)$ is a projective lattice if and only if for each $p \in P$ there are two finite subsets $A(p) \subseteq$ $\{q \in P: q \geqq p\}$ and $B(p)=\{q \in P: q \leqq p\}$ such that if $p \leqq q$ in $P$, then $A(p) \cap B(q) \neq \varnothing$.

This notation and terminology differs from that of Dean [7] and Dilworth [8], where the term "completely free lattice generated by $\boldsymbol{P}$ " is used. 
Let $P_{0}$ be the partially ordered set consisting of two antichains $C$ and $D$ such that for all $c \in C$ and $d \in D, c \leqq d$, and $D$ is finite. Let $P_{1}$ be similar to $P_{0}$ except now $C$ is uncountable and $D$ is infinite. Let $P_{2}=C \cup E$, where $C$ is an uncountable antichain and $E$ is isomorphic to the dual of $\omega$, and $c \leqq e$ for all $c \in C$ and $e \in E$. Let $P_{3}$ be $P_{1} \cup\{r\}$ such that for $c \in C$ and $d \in D, c \leqq r \leqq d$. Let $P_{4}$ be the partially ordered set consisting of the atoms and coatoms of the Boolean algebra $2^{\boldsymbol{\aleph}_{1}}$. Of course, $F L\left(P_{0}\right)$ is the ordinal sum of two free lattices, one of them finitely generated. $F L\left(P_{1}\right)$ is the ordinal sum of two infinitely generated free lattices, one of them uncountable. $F L\left(P_{3}\right)$ is $F L\left(P_{1}\right)$ with a "middle" element adjoined. $F L\left(P_{2}\right)$ is a free lattice with a copy of the dual of $\omega$ on top if it. Using the corollary we can see that $F L\left(P_{0}\right)$ and $F L\left(P_{3}\right)$ are projective, but that $F L\left(P_{1}\right), F L\left(P_{2}\right)$ and $F L\left(P_{4}\right)$ are not. To see that $F L\left(P_{0}\right)$ is projective, let $A(c)=D \cup\{c\}, B(c)=\{c\}, A(d)=\{d\}, B(d)=$ $\{d\}$ for all $c \in C, d \in D$. For $F L\left(P_{3}\right)$ let $A(c)=\{c, r\}, B(c)=\{c\}$, $A(r)=\{r\}=B(r), A(d)=\{d\}$, and $B(d)=\{d, r\}$.

To see that $F L\left(P_{1}\right)$ is not projective, suppose sets $A(p)$ and $B(p)$ exist as in the corollary. Then $A(c)$ consists of finitely many elements of $D$ and possibly $c$ itself. Let $C_{i}=\{c \in C:|A(c)| \leqq i\}$. Then $C=$ $\cup_{i<\omega} C_{i}$. Since $C$ is uncountable, there is a $k$ such that $C_{k}$ is uncountable. Since $D$ is infinite, there are $k+1$ distinct elements, $d_{1}, \cdots, d_{k+1}$ in $D$. Since $B\left(d_{1}\right) \cup \cdots \cup B\left(d_{k+1}\right)$ is a finite set, $C_{k}^{\prime}=$ $C_{k}-\left(B\left(d_{1}\right) \cup \cdots \cup B\left(d_{k+1}\right)\right)$ is still infinite. If $c \in C_{k}^{\prime}$, then $c \leqq d_{i}, i=$ $1, \cdots, k+1$. Thus $A(c) \cap B\left(d_{i}\right) \neq \varnothing$. By the definition of $C_{k}^{\prime}$, $c \notin B\left(d_{i}\right)$. This forces $d_{i} \in A(c), \quad i=1, \cdots, k+1$, countradicting $|A(c)| \leqq k . \quad$ A similar argument can be employed to show that $F L\left(P_{2}\right)$ is not projective. In this case we define $C_{e}=\{c \in C: x \in A(c) \cap E$ implies $x \geqq e\}$.

For $i=0,1,2,3,4, F L\left(P_{i}\right)$ is a countable union of antichains, and hence, contains no uncountable chain. For $i=0,1,2,3$, this is obvious from the descriptions of $F L\left(P_{1}\right)$ given above; for $F L\left(P_{4}\right)$, one can modify the arguments of Galvin and Jónsson [9]. These examples show that condition (4) of Theorem 1 cannot be replaced by (8), or even the stronger condition of being the countable union of antichains. If $P$ is the partially ordered set of [6] p. 5 cited above, then $F L(P)$ has no uncountable chains by Theorem 1 of [20]. This gives a counter-example to (8) but not the stronger condition mentioned above.

Although $F L\left(P_{1}\right)$ is not projective, $P_{1}$ is isomorphic to a subpartially ordered set of a free lattice, since $P_{1} \subseteq P_{3}$ and $F L\left(P_{3}\right)$ is projective. Similarly $P_{2}$ is embeddable in a free lattice. (In fact it is not hard to show that $F L\left(P_{1}\right)$ and $F L\left(P_{2}\right)$ are both sublattices of a free lattice.) Arguments similar to those above show that $F L\left(P_{4}\right)$ is not projective. We shall show that $P_{4}$ is not even order-embeddable in a free 
lattice. Let $P_{4}=\left\{a_{i}: i \in I\right\} \cup\left\{b_{i}: i \in I\right\}$ where $|I|=\boldsymbol{N}_{1}$, and $a_{i}<b_{j}$ for all $i$ and $j$ with $i \neq j$. Suppose $P_{4}$ is order-embedded into a free lattice, $F L(X)$. Let $G$ be the group of those automorphisms of $F L(X)$ induced from permutation of $X$. Define an equivalence relation on $I$ by $i \sim j$ if there is a $\sigma \in G$ such that $\sigma\left(a_{i}\right)=a_{j}$ and $\sigma\left(b_{i}\right)=b_{j}$ (cf. [9]). Intuitively, $i \sim j$ means that $a_{i}$ and $a_{j}$ may be represented with the same lattice term except for a change in the variables, and the same statement holds for $b_{i}$ and $b_{j}$ with the same change of variables. With the aid of this, the reader can show that the relation $\sim$ has only countably many classes. Consequently, one of these classes, $I^{\prime}$, must be uncountable. Of course, $P_{4}^{\prime}=\left\{a_{i}: i \in I^{\prime}\right\} \cup\left\{b_{i}: i \in I^{\prime}\right\}$ is isomorphic to $P_{4}$. Thus, if $P_{4}$ is order-embeddable into $F L(X)$, then there exist $u$ and $v \in F L(X)$ and $\sigma_{i} \in G, i \in I$, such that $a_{i}=\sigma_{i}(v)$ and $b_{i}=\sigma_{i}(u)$.

Let $x_{i}, \cdots, x_{n}$ be the distinct variables which occur in either the canonial form of $u$ or of $v$. Consider the largest integer $k$ such that for some $i \in I$ and uncountably many $j \in I$

$$
\left|\left\{\sigma_{i}\left(x_{1}\right), \cdots, \sigma_{i}\left(x_{n}\right)\right\} \cap\left\{\sigma_{j}\left(x_{1}\right), \cdots, \sigma_{j}\left(x_{n}\right)\right\}\right|=k .
$$

Let $i_{0}$ be such an $i$ and let $I^{\prime} \subseteq I$ be those $j$ 's for which the above equation holds. If we let $y_{1}=\sigma_{i_{0}}\left(x_{1}\right), \cdots, y_{n}=\sigma_{i 0}\left(x_{n}\right)$, then $\sigma_{j}\left(x_{t}\right)=\sigma_{j} \sigma_{i_{0}}^{-1}\left(y_{t}\right), t=$ $1, \cdots, n$ : Let $\tau_{j}=\sigma_{j} \sigma_{i_{0}}^{-1}$ and $r=\sigma_{i 0}(u), \quad s=\sigma_{i o}(v)$. Then $\tau_{j}(r)=$ $\sigma_{j}(u)$. We make two additional reductions. First, there is an uncountable subset $I^{\prime \prime}$ of $I^{\prime}$ such that

$$
\left\{y_{1}, \cdots, y_{n}\right\} \cap\left\{\tau_{i}\left(y_{1}\right), \cdots, \tau_{i}\left(y_{n}\right)\right\}=\left\{y_{1}, \cdots, y_{n}\right\} \cap\left\{\tau_{j}\left(y_{1}\right), \cdots, \tau_{j}\left(y_{n}\right)\right\}
$$

for each $i, j \in I^{\prime \prime}$. Thus, we may assume $\left\{y_{1}, \cdots, y_{n}\right\} \cap$ $\left\{\tau_{i}\left(y_{1}\right), \cdots, \tau_{i}\left(y_{n}\right)\right\}=\left\{y_{1}, \cdots, y_{k}\right\}$, for each $i \in I^{\prime \prime}$. Let $h_{i}:\{1, \cdots, k\}$ $\rightarrow\{1, \cdots, n\}$ be defined by $y_{t}=\tau_{i}\left(y_{h_{i}(t)}\right), t=1, \cdots, k, i \in I^{\prime \prime}$. That is, $h_{i}(t)$ is the position where $y_{t}$ occurs in $\tau_{i}\left(y_{1}\right), \cdots, \tau_{t}\left(y_{n}\right)$. Again, there is an uncountable subset $I^{\prime \prime \prime}$ of $I^{\prime \prime}$ such that $h_{i}=h_{j}$ for each $i, j \in I^{\prime \prime \prime}$. Hence, we may assume that $h_{i}(t)=t, t=1, \cdots, k, i \in I^{\prime \prime \prime}$. By the definition of $k$, there are $i$ and $j$ in $I^{\prime \prime \prime}, \quad i \neq j$, such that $\left\{\tau_{i}\left(y_{1}\right), \cdots, \tau_{i}\left(y_{n}\right)\right\} \cap$ $\left\{\tau_{j}\left(y_{1}\right), \cdots, \tau_{j}\left(y_{n}\right)\right\}=\left\{y_{1}, \cdots, y_{k}\right\}$. Thus,

and

$$
\left\{\tau_{i}\left(y_{1}\right), \cdots, \tau_{i}\left(y_{n}\right)\right\}=\left\{y_{1}, \cdots, y_{k}, z_{k+1}, \cdots, z_{n}\right\}
$$

$$
\left\{\tau_{j}\left(y_{1}\right), \cdots, \tau_{j}\left(y_{n}\right)\right\}=\left\{y_{1}, \cdots, y_{k}, z_{k+1}^{\prime}, \cdots, z_{n}^{\prime}\right\}
$$

where $\left\{z_{k+1}^{\prime}, \cdots, z_{n}^{\prime}\right\} \cap\left\{y_{1}, \cdots, y_{k}, z_{k+1}, \cdots, z_{n}\right\}=\varnothing$. Now let $f$ be the endomorphism of $F L(X)$ extending the map $f\left(z_{k+1}^{\prime}\right)=z_{k+1}, \cdots, f\left(z_{n}^{\prime}\right)=z_{n}$, 
and $f(x)=x$ for all $x \in X-\left\{z_{k+1}^{\prime}, \cdots, z_{n}^{\prime}\right\}$. Now

$$
\begin{aligned}
f\left(\tau_{j}\left(r\left(y_{1}, \cdots, y_{n}\right)\right)\right) & =f\left(r\left(\tau_{j}\left(y_{1}\right), \cdots, \tau_{j}\left(y_{n}\right)\right)\right)=f\left(r\left(y_{1}, \cdots, y_{k}, z_{k+1}^{\prime}, \cdots, z_{n}^{\prime}\right)\right) \\
& =r\left(y_{1}, \cdots, y_{k}, z_{k+1}, \cdots, z_{n}\right)=\tau_{i}\left(r\left(y_{1}, \cdots, y_{n}\right)\right) .
\end{aligned}
$$

On the other hand, it is easy to see that $f\left(\tau_{i}(s)\right)=\tau_{i}(s)$. Since $i \neq j$, $\tau_{i}(s) \leqq \tau_{j}(r)$ and thus $\tau_{i}(s)=f\left(\tau_{i}(s)\right) \leqq f\left(\tau_{j}(r)\right)=\tau_{i}(r)$. But $\tau_{i}(s)$ and $\tau_{i}(r)$ are images under the automorphism $\tau_{i} \sigma_{i_{0}}=\sigma_{l}$ of $v$ and $u$, respectively. However, since $u$ and $v$ are incomparable, this implies $\tau_{i}(s)$ and $\tau_{i}(r)$ are incomparable, a contradiction.

4. Projective distributive lattices. The definition of projective distributive lattices is analogous to that of projective lattices except, of course, distributive lattices are used. Since epimorphisms are not onto in the category of distributive lattices, this definition is different from the categorical one (cf. [3], where the term weakly projective is used). Projective distributive lattices have been characterized by Balbes and Horn [2], [4]. The following theorem is a slight improvement of their theorem.

THEOREM 4. A distributive lattice $L$ is projective if and only if every element of $L$ is a finite join of join-irreducible elements and a finite meet of meet-irreducible elements, the meet of two join-irreducible elements is join-irreducible, and L satisfies condition (4) of Theorem 1.

Proof. It was shown in [3], that this theorem holds if (4) is replaced by the condition that there is a homomorphism of a free distributive lattice $F D(X)$ onto $L$, which has an order-preserving transversal. By an argument similar to the proof of Theorem 2, one can see that this latter condition is equivalent to (4).

The conditions of Theorem 1 are related to conditions of Theorem 4. For example, in a distributive lattice $\cup D_{k}(L)=D_{1}(L)$. Thus $\cup D_{k}(L)=L$ holds in a distributive lattice if and only if every element of $L$ is a finite join of join-irreducibles (which are the same as the join-primes in a distributive lattice).The condition $(W)$ implies that the join-irreducibles of $L$ are closed under finite meets. Furthermore, in a distributive lattice condition (3) of Theorem 1 holds if and only if $D_{1}(L)=L$. Also note the examples of the last section can be modified so as to apply to the distributive case.

Finally, we point out that condition (4) alone characterizes projective Boolean algebras. Thus this result, which will be presented in a subsequent note, generalizes the result of Halmos [12] that every countable Boolean algebra is projective. 


\section{REFERENCES}

1. K. Baker and A. Hales, Distributive projective lattices, Canad. J. Math., 22 (1970), 472-475.

2. R. Balbes, Projective and injective distributive lattices, Pacific J. Math., 21 (1967), 405-420.

3. R. Balbes and P. Dwinger, Distributive Lattices, University of Missouri Press, Columbia, MO, 1974.

4. R. Balbes and A. Horn, Projective distributive lattices, Pacific J. Math., 33 (1970), 273-279.

5. P. Crawley and R. A. Dean, Free lattices with infinite operations, Trans. Amer. Math. Soc., 92 (1959), 35-47.

6. P. Crawley and R. P. Dilworth, Algebraic Theory of Lattices, Prentice-Hall, Englewood Cliffs, NJ, 1973.

7. R. A. Dean, Completely free lattices generated by partially ordered sets, Trans. Amer. Math. Soc., 83 (1956), 238-249.

8. R. P. Dilworth, Lattices with unique complements, Trans. Amer. Math. Soc., 57 (1945), 123-154.

9. F. Galvin and B. Jónsson, Distributive sublattices of a free lattice, Canad. J. Math., 13 (1961), 265-272.

10. H. Gaskill and C. Platt, Sharp transferability and finite sublattices of free lattices, Canad. J. Math., 27 (1975), 1036-1041.

11. G. Grätzer, Lattice Theory: First Concepts and Distributive Lattices, W. H. Freeman Co., San Francisco, CA, 1971.

12. P. Halmos, Injective and projective Boolean algebras, Lattice Theory, Proceedings of Symposia in Pure Math., 2 Amer. Math. Soc., Providence, RI, 1961.

13. B. Jónsson, Sublattices of a free lattice, Canad. J. Math., 13 (1961), 256-264.

14. B. Jónsson and J. Kiefer, Finite sublattices of a free lattice, Canad. J. Math., 14 (1962), 487-497.

15. B. Jónsson and J. B. Nation, A report on sublattices of a free lattice, to appear in Contributions to Universal Algebra, North-Holland.

16. A. Kostinsky, Projective lattices and bounded homomorphisms, Pacific J. Math., 40 (1972), 111-119.

17. R. McKenzie, Equational bases and nonmodular lattice varieties, Trans. Amer. Math. Soc., 174 (1972), 1-43.

18. P. Whitman, Free lattices, Annals of Math., 42 (1941), 325-330.

19. - Free lattices II, Annals of Math., 43 (1942), 104-115.

20. M. Adams and D. Kelly, Chain conditions in free products of lattices, Algebra Universalis, 7 (1977), 245-258.

Received November 23, 1976. This research was supported in part by NSF Grants Nos. MPS 73-08589 A02 and GP-29129-A3.

UNIVERSITY OF HAWAII

HoNOLULU, HI 96822

AND

VANDERBILT UNIVERSITY

NASHVILLE, TN 37235 


\section{PACIFIC JOURNAL OF MATHEMATICS EDITORS}

ICHARD ARENS (Managing Editor)

niversity of California

os Angeles, CA 90024

\section{. A. BEAUmont}

niversity of Washington

sattle, WA 98105

. C. MOORE

niversity of California

erkeley, CA 94720
J. DUGUNDJI

Department of Mathematics

University of Southern California

Los Angeles, CA 90007

R. FINN AND J. MiLgRAM

Stanford University

Stanford, CA 94305

\section{ASSOCIATE EDITORS}
F. BECKENBACH
B. H. NEUMANN
F. WOLF
K. YosHidA

\section{SUPPORTING INSTITUTIONS}

NIVERSITY OF BRITISH COLUMBIA

UNIVERSITY OF SOUTHERN CALIFORNIA

ALIFORNIA INSTITUTE OF TECHNOLOGY STANFORD UNIVERSITY

NIVERSITY OF CALIFORNIA

ONTANA STATE UNIVERSITY

UNIVERSITY OF HAWAII

NIVERSITY OF NEVADA

UNIVERSITY OF TOKYO

EW MEXICO STATE UNIVERSITY

UNIVERSITY OF UTAH

REGON STATE UNIVERSITY

NIVERSITY OF OREGON

WASHINGTON STATE UNIVERSITY

UNIVERSITY OF WASHINGTON

SAKA UNIVERSITY

AMERICAN MATHEMATICAL SOCIETY

The Supporting Institutions listed above contribute to the cost of publication of this Journal, but they a t owners or publishers and have no responsibility for its contents or policies.

Mathematical papers intended for publication in the Pacific Journal of Mathematics should be in typ Irm or offset-reproduced (not dittoed), double spaced with large margins. Underline Greek letters in re ierman in green, and script in blue. The first paragraph or two must be capable of being used separately as 'nopsis of the entire paper. Items of the bibliography should not be cited there unless absolutely necessary, hich case they must be identified by author and Journal, rather than by item number. Manuscripts, uplicate, may be sent to any one of the four editors. Please classify according to the scheme of Math. Revieu Idex to Vol. 39. All other communications should be addressed to the managing editor, or Elaine Bart Iniversity of California, Los Angeles, California, 90024.

100 reprints are provided free for each article, only if page charges have been substantially pai dditional copies may be obtained at cost in multiples of 50 .

The Pacific Journal of Mathematics is issued monthly as of January 1966. Regular subscription rate: \$72. year (6 Vols., 12 issues). Special rate: $\$ 36.00$ a year to individual members of supporting institutions.

Subscriptions, orders for numbers issued in the last three calendar years, and changes of address should ent to Pacific Journal of Mathematics, 103 Highland Boulevard, Berkeley, California, 94708.

UBLISHED BY PACIFIC JOURNAL OF MATHEMATICS, A NON-PROFIT CORPORATION

Printed at Jerusalem Academic Press, POB 2390, Jerusalem, Israel.

Copyright (C) 1978 Pacific Journal of Mathematics

All Rights Reserved 


\section{Pacific Journal of Mathematics}

\section{Vol. 75, No. $1 \quad$ September, 1978}

Mieczyslaw Altman, General solvability theorems

Denise Amar and Eric Amar, Sur les suites d'interpolation en plusieurs variables ..........................................

Herbert Stanley Bear, Jr. and Gerald Norman Hile, Algebras which satisfy a second order linear partial differential equation ..................

Marilyn Breen, Sets in $R^{d}$ having $(d-2)$-dimensional kernels ............

Gavin Brown and William Moran, Analytic discs in the maximal ideal space

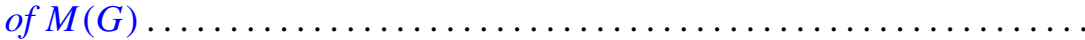

Ronald P. Brown, Quadratic forms with prescribed Stiefel-Whitney

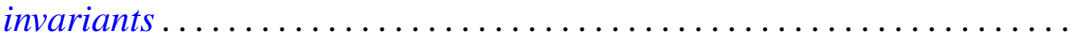

Gulbank D. Chakerian and H. Groemer, On coverings of Euclidean space by convex sets

S. Feigelstock and Z. Schlussel, Principal ideal and Noetherian groups.....

Ralph S. Freese and James Bryant Nation, Projective lattices ............

Harry Gingold, Uniqueness of linear boundary value problems for

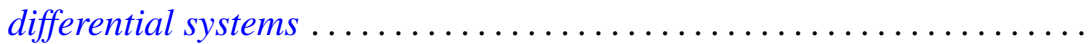

John R. Hedstrom and Evan Green Houston, Jr., Pseudo-valuation domains. . .

William Josephson, Coallocation between lattices with applications to measure extensions

M. Koskela, A characterization of non-negative matrix operators on $l^{p}$ to $l^{q}$ with $\infty>p \geq q>1$

Kurt Kreith and Charles Andrew Swanson, Conjugate points for nonlinear differential equations...........................

Shoji Kyuno, On prime gamma rings ........................ 185

Alois Andreas Lechicki, On bounded and subcontinuous multifunctions ..

Roberto Longo, A simple proof of the existence of modular automorphisms in approximately finite-dimensional von Neumann algebras ...

Kenneth Millett, Obstructions to pseudoisotopy implying isotopy for

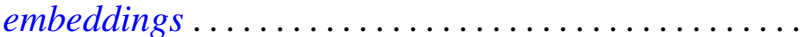

William F. Moss and John Piepenbrink, Positive solutions of elliptic equations. .

Mitsuru Nakai and Leo Sario, Duffin's function and Hadamard's

conjecture

Mohan S. Putcha, Word equations in some geometric semigroups ...

Walter Rudin, Peak-interpolation sets of class $C^{1} \ldots \ldots \ldots$

Elias Saab, On the Radon-Nikodým property in a class of locally convex

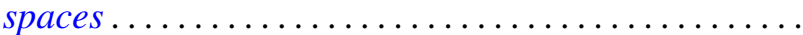

